\title{
Fuzzy sliding-mode control with low pass filter to reduce chattering effect: an experimental validation on Quanser SRIP
}

\author{
S BALAMURUGAN $^{1, *}$, P VENKATESH $^{2}$ and M VARATHARAJAN ${ }^{2}$ \\ ${ }^{1}$ Department of Electronics and Instrumentation Engineering, K.L.N. College of Engineering, Madurai, India \\ ${ }^{2}$ Department of Electrical and Electronics Engineering, Thiagarajar College of Engineering, Madurai, India \\ e-mail: sbalan18@yahoo.com; pveee@tce.edu; varatharajan@tce.edu
}

MS received 16 July 2015; revised 6 December 2016; accepted 31 January 2017; published online 13 October 2017

\begin{abstract}
Stabilization of a single rotary inverted pendulum (SRIP) system is a benchmark nonlinear control problem. Inherent instability of SRIP in upright position and the presence of modelling uncertainty pose a challenge for control engineers. Sliding-mode control (SMC) is the most widely accepted robust control technique. Various versions of SMCs to stabilize SRIP have been reported in literature. Advantages of SMC come at the cost of chattering of input, which inhibits its usage in many practical applications. Several works have been done to reduce the chattering in SMC. Application of fuzzy logic in SMC to reduce chattering has been analysed extensively in the literature. Even though implementation of fuzzy SMC (FSMC) in theory/simulations completely eliminates chattering, practical implementation of FSMC does not reduce chattering to zero. In this paper, a filtered FSMC is proposed, which filters the high frequency component in the system input. Experimental results of SMC, FSMC and a fuzzy sliding-mode controller with filter (FSMCF) are given in this paper. Results indicate that filtering the output of FSMCF reduces the chattering effect in the system without affecting the closed loop dynamics.
\end{abstract}

Keywords. Chattering reduction; sliding-mode control (SMC); fuzzy sliding-mode control (FSMC); fuzzy sliding-mode controller with filter (FSMCF); low pass filter (LPF); single rotary inverted pendulum (SRIP).

\section{Introduction}

The single rotary inverted pendulum (SRIP) is a widely used nonlinear system due to its static instability. SRIP system is a benchmark problem in nonlinear dynamic control. It is commonly used to verify the robustness of any proposed nonlinear controller. The SRIP has nonlinear characteristics and it is easy to analyse systems near the equilibrium point. For these concepts, many researchers have used this typical system to verify their ideas. The problem of stabilizing an unstable system occurs in practice in the areas of missile stabilization, control of aerospace rocket during take-off and control of robotics. The energy-based swing-up control proposed for a general inverted pendulum [1] is performed only in simulation. The Full-State Feedback Control (FSFC) and Linear-Quadratic Regulator (LQR) control are used for stabilization of an inverted pendulum by Akhtaruzzaman and Shafie [2] and they are not applied to real-time set-ups. Some researchers have approached the nonlinear problem by simulations [3]. An approximate input-state linearization method is discussed by Chen et al [4] but uncertainty disturbances are not considered. A fuzzy rule controller based on single-input module is presented, where each input term is

*For correspondence assigned to a Single Input Rule Module (SIRM) [5, 6]. A fuzzy swing-up control strategy has been proposed [7], where the least preference is given for stabilization control.

The sliding-mode control (SMC) methodologies emerged as an effective tool to tackle uncertainty and disturbances, which are inevitable in most of the practical systems [8]. SMC is a particular class of variable structure control, which was introduced by Utkin [9] and his colleagues. SMC is a robust nonlinear feedback control method $[10,11]$, but the drawback is undesirable chattering problem, which causes poor control accuracy, high oscillation of mechanical parts and heat loss in power electrical circuit. The solution of the chattering reduction problem is of great importance when exploiting the benefit of sliding-mode controller [12]. The chattering phenomenon is due to three main causes, namely unmodelled dynamics, switching gain value and discontinuous function in SMC. Even in SMC design, these causes are to taken into account, which minimizes the chattering effect $[13,14]$.

SMC has been used for swing-up and stabilization of a rotary inverted pendulum (RIP) by Park and Chwa [15]. They concentrate more on settling time and stabilization of RIP at minimal time. However, the response is not underdamped and hence it affects the mechanical component of the system [6]. Adaptive fuzzy sliding-mode control (FSMC) is a robust control approach [5], which performs 
conventional PID and SMC. SMC signal filtered using a low pass filter (LPF) is discussed by Park and Lee [16]. In addition, in the steady state, chattering effect appears as a high switching frequency oscillation near the desired equilibrium point [17]. Since it is almost always desirable to avoid chattering effect, many researchers have performed studies to overcome this phenomenon by dynamic sliding-mode control (DSMC) [18]. Similarly, chattering reduction of an induction motor has been analysed by Tseng and Chen [19].

To overcome these difficulties and to reduce effect of chattering in a nonlinear system in linear operating region, a new methodology is proposed. FSMC with and without filter for stabilization of SRIP is proposed in this paper. The application of the proposed method is sensitive to parameter variation and external disturbances. The implementation of this scheme to SRIP in real time improves the stabilization performance and reduces the chattering effect, while it smoothens swing-up and provides stable control of highly complex nonlinear systems. This paper is organized as follows. SRIP prototype explanation is given in section 2. Section 3 describes the mathematical model of SRIP system in state space domain. Section 4 provides the development of SMC, and FSMC with and without filter. A real-time experimental set-up and its associated hardware and DAQ cards are presented in section 5. Section 6 illustrates the implementation results with and without filter for stabilization control of SRIP in both time and frequency domains. Finally, the conclusion is provided in section 7.

\section{SRIP}

The SRIP, which is shown in figure 1, is driven by a Quanser make rotary DC servo motor system [20]. The DC servo motor drives an independent output gear, whose angular position is measured by an encoder. The inverted pendulum arm is mounted on the output gear. The inverted pendulum is attached to a hinge instrumented with another encoder at the end of the pendulum arm. This second encoder measures the angular displacement of the pendulum. The SRIP is interfaced by a Quanser make Q8 USB data acquisition card and driven by Matlab/simulink-based real time QuaRC software (www.qaunser.com). The inverted pendulum has two equilibrium points: stable and unstable. At the stable equilibrium point the aluminium rod is in vertical position and pointing down, while at the unstable equilibrium point the aluminium rod is in vertical position and pointing up.

\section{Mathematical model of SRIP system}

A schematic of SRIP is shown in figure $2 ; \alpha_{1}$ and $\theta_{1}$ are employed as the generalized coordinates (table 1) to describe the inverted pendulum system. The pendulum is

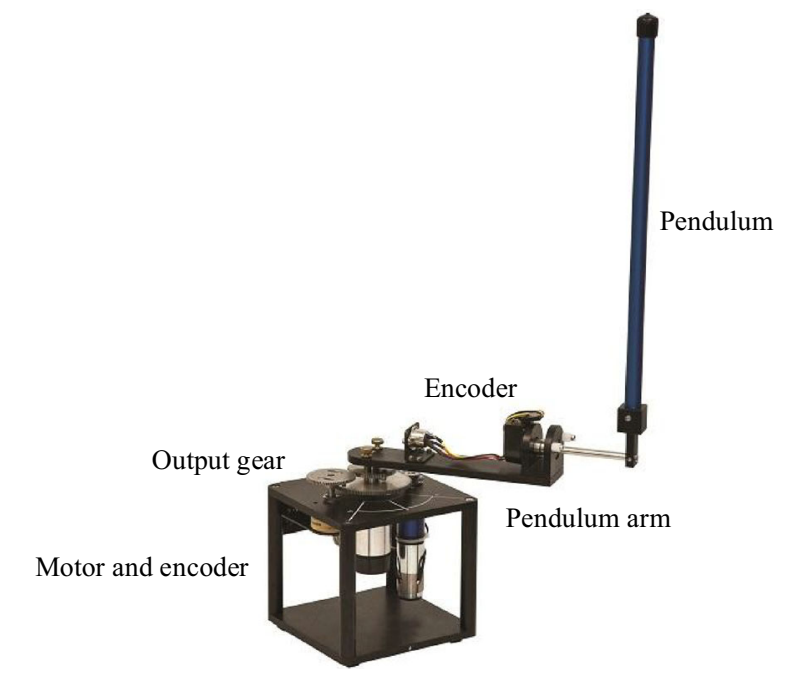

Figure 1. Single rotary inverted pendulum.
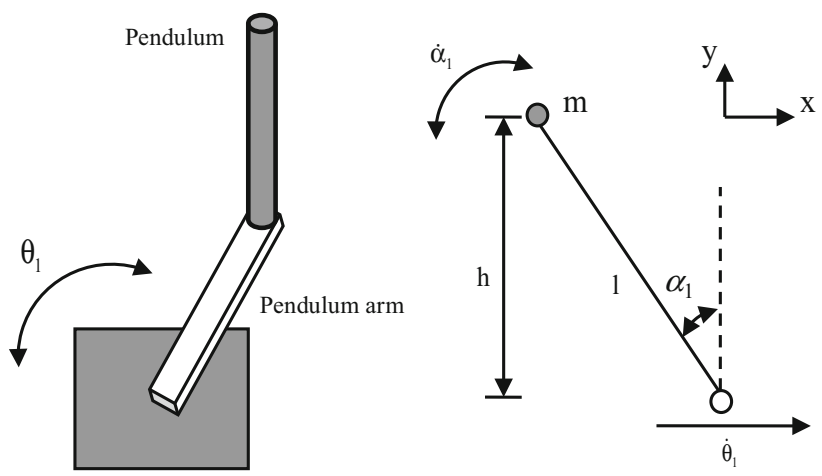

Figure 2. Schematic of a single rotary inverted pendulum.

displaced by an angle $\alpha_{1}$ while the direction of $\theta_{1}$ is in the $x$-direction. The mathematical model can be derived by examining the velocity of the pendulum at the centre of mass.

Velocity of pendulum lumped mass element is given by

$$
v=-l \cos \alpha_{1}\left(\dot{\alpha}_{1}\right) x+l \sin \alpha_{1}\left(\dot{\alpha}_{1}\right) y .
$$

The only potential energy in the system is gravity and it is given by

$$
V=m g l \cos \alpha_{1} .
$$

The pendulum arm also moves horizontally at a rate of

$$
V_{\text {arm }}=r \dot{\theta}_{1}
$$

Equations (1) and (2) are resolved to $x$ and $y$ velocity components as follows:

$$
\begin{gathered}
v_{x}=r \dot{\theta}_{1}-l \cos \alpha_{1}\left(\dot{\alpha}_{1}\right), \\
v_{y}=-l \sin \alpha_{1}\left(\dot{\alpha}_{1}\right) .
\end{gathered}
$$


Table 1. Nomenclature of the SRIP.

\begin{tabular}{lc}
\hline Symbol & Description \\
\hline$\alpha_{1}$ & Pendulum position \\
$\dot{\alpha}_{1}$ & Pendulum velocity \\
$\ddot{\alpha}_{1}$ & Pendulum acceleration \\
$\theta_{1}$ & Servo motor gear position \\
$\dot{\theta}_{1}$ & Servo motor gear velocity \\
$\ddot{\theta}_{1}$ & Servo motor gear acceleration \\
$\eta_{g r}$ & Gearbox efficiency \\
$\eta_{m r}$ & Motor efficiency \\
$B_{e q}$ & Equivalent viscous damping coefficient \\
$h$ & Gravity acceleration \\
$g$ & Pendulum inertia about its centre of mass \\
$J_{c m}$ & Equivalent moment of inertia at the load \\
$J_{e q}$ & Motor inertia (kg m $\left.{ }^{2}\right)$ \\
$J_{m r}$ & Total gear ratio (kg) \\
$K_{g r}$ & Back-EMF constant \\
$K_{m r}$ & Motor torque constant (Nm/A) \\
$K_{t}$ & Length of pendulum (m) \\
$l$ & Pendulum mass (kg) \\
$m$ & Rotating arm length (m) \\
$r$ & Motor armature resistance $(\Omega)$ \\
$R_{m r}$ & Output torque of DC motor (Nm) \\
$T_{\text {output }}$ & Velocity of pendulum centre of mass in the $x$-direction \\
$V_{x}$ & Velocity of pendulum centre of mass in the $y$-direction \\
$V_{y}$ & \\
$V_{m r}$ & \\
\hline &
\end{tabular}

The kinetic energy in the system arises from the moving servo motor, the velocity of the point mass in $x$-direction, the velocity of the point mass in the $y$-direction and the rotating pendulum about its centre of mass. Hence, the total kinetic energy is

$$
T=K E_{\text {motor }}+K E_{v x}+K E_{v y}+K E_{\text {pendulum }} .
$$

Using all the information, the kinetic energy can be represented as follows:

$$
\begin{aligned}
T= & \frac{1}{2} J_{e q} \dot{\theta}_{1}^{2}+\frac{1}{2} m\left[\left(r \dot{\theta}_{1}-l \cos \alpha_{1}\left(\dot{\alpha}_{1}\right)\right)^{2}+\left(-l \sin \alpha_{1}\left(\dot{\alpha}_{1}\right)\right)^{2}\right] \\
& +\frac{1}{2} J_{c m} \dot{\alpha}_{1}^{2}, \\
T & =\frac{1}{2}\left(J_{e q}+m r^{2}\right) \dot{\theta}_{1}^{2}+\frac{2}{3} m l^{2} \dot{\alpha}_{1}^{2}-\frac{1}{2}\left[m l r \cos \alpha_{1}\left(\dot{\theta}_{1}\right)\left(\dot{\alpha}_{1}\right)\right] .
\end{aligned}
$$

The Lagrangian can be formulated as follows:

$$
L=T-V
$$

$$
\begin{aligned}
L= & \frac{1}{2}\left(J_{e q}+m r^{2}\right) \dot{\theta}_{1}^{2}+\frac{2}{3} m l^{2} \dot{\alpha}_{1}^{2}-\frac{1}{2} m l r \cos \alpha_{1}\left(\dot{\theta}_{1}\right)\left(\dot{\alpha}_{1}\right) \\
& -m g l \cos \alpha_{1} .
\end{aligned}
$$

Considering the generalized coordinates as $\theta_{1}$ and $\alpha_{1}$, the Euler-Lagrange equations are

$$
\begin{gathered}
\frac{d}{d t}\left(\frac{\partial L}{\partial \dot{\theta}_{1}}\right)-\frac{\partial L}{\partial \theta_{1}}=T_{\text {output }}-B_{e q} \dot{\theta}_{1}, \\
\frac{d}{d t}\left(\frac{\partial L}{\partial \dot{\alpha}_{1}}\right)-\frac{\partial L}{\partial \alpha_{1}}=0 .
\end{gathered}
$$

Substituting the relevant values gives

$$
\begin{aligned}
& \frac{d}{d t}\left(J_{e q} \dot{\theta}_{1}+m r^{2} \dot{\theta}_{1}-m r l \cos \alpha_{1}\left(\dot{\alpha}_{1}\right)\right)-0=T_{\text {output }}-B_{\text {eq }} \dot{\theta}_{1}, \\
& J_{\text {eq }} \ddot{\theta}_{1}+m r^{2} \ddot{\theta}_{1}-m r l \cos \alpha_{1}\left(\ddot{\alpha}_{1}\right)+m r l \sin \alpha_{1}\left(\dot{\alpha}_{1}^{2}\right) \\
& \quad=T_{\text {output }}-B_{\text {eq }} \dot{\theta}_{1} .
\end{aligned}
$$

Neglecting the higher order terms and simplifying yields

$$
\begin{gathered}
J_{e q} \ddot{\theta}_{1}+m r^{2} \ddot{\theta}_{1}-m r l \cos \alpha_{1}\left(\ddot{\alpha}_{1}\right)=T_{\text {output }}-B_{e q} \dot{\theta}_{1}, \\
-m l r \cos \alpha_{1}\left(\ddot{\theta}_{1}\right)+\frac{4}{3} m l^{2} \ddot{\alpha}_{1}-m g l \sin \alpha_{1}=0 .
\end{gathered}
$$

These complex nonlinear coupled equations constitute an underactuated system.

There are two equilibrium points: $\alpha_{1}=\pi$ at which pendulum is down in stable position and $\dot{\alpha}_{1}=0$ at which pendulum is up in unstable position. The linearized model about the unstable position is

$$
\begin{aligned}
\left(J e q+m r^{2}\right) \ddot{\theta}_{1}-m l r \ddot{\alpha}_{1}+B_{e q} \dot{\theta}_{1} & =T_{\text {output }}, \\
-m l r \ddot{\theta}_{1}+\frac{4}{3} m l^{2} \ddot{\alpha}_{1}-m g l \alpha_{1} & =0 .
\end{aligned}
$$

The output torque of the servo motor, which acts on the load, is defined as

$$
T_{\text {output }}=\frac{\eta_{g r} \eta_{m r} K_{g r} K_{t}\left(V_{m r}-K_{g r} K_{m r} \dot{\theta}\right)}{R_{m r}} .
$$

The state space representation of the complete system is

$$
\begin{gathered}
{\left[\begin{array}{c}
\dot{\theta}_{1} \\
\dot{\alpha}_{1} \\
\ddot{\theta}_{1} \\
\ddot{\alpha}_{1}
\end{array}\right]=\left[\begin{array}{cccc}
0 & 0 & 1 & 0 \\
0 & 0 & 0 & 1 \\
0 & \frac{b d}{E_{1}} & -\frac{c G}{E_{1}} & 0 \\
0 & \frac{a d}{E_{1}} & -\frac{b G}{E_{1}} & 0
\end{array}\right]\left[\begin{array}{c}
\theta_{1} \\
\alpha_{1} \\
\dot{\theta}_{1} \\
\dot{\alpha}_{1}
\end{array}\right]+\left[\begin{array}{c}
0 \\
0 \\
\frac{c f}{E_{1}} \\
\frac{b f}{E_{1}}
\end{array}\right] V_{m r},} \\
a=J_{e q}+m r^{2} \\
b=m l r \\
c=\frac{4}{3} m l^{2}, \\
d=m g l, \\
E_{1}=a c-b^{2} \\
f=\frac{\eta_{m r} \eta_{g r} K_{t} K_{g r}}{R_{m r}} \\
G=\frac{\eta_{m r} \eta_{g r} K_{g r}^{2} K_{m r} K_{t}+B_{e q} R_{m r}}{R_{m r}}
\end{gathered}
$$


Substituting the values of the parameters specified in the appendix (table 3), the state space model for SRIP is obtained as follows:

$$
\begin{aligned}
{\left[\begin{array}{c}
\dot{\theta}_{1} \\
\dot{\alpha}_{1} \\
\ddot{\theta}_{1} \\
\ddot{\alpha}_{1}
\end{array}\right]=} & {\left[\begin{array}{cccc}
0 & 0 & 1 & 0 \\
0 & 0 & 0 & 1 \\
0 & 55.9258 & -18.9606 & 0 \\
0 & 97.2622 & -18.1705 & 0
\end{array}\right]\left[\begin{array}{c}
\theta_{1} \\
\alpha_{1} \\
\dot{\theta}_{1} \\
\dot{\alpha}_{1}
\end{array}\right] } \\
& +\left[\begin{array}{c}
0 \\
0 \\
33.2158 \\
31.8318
\end{array}\right] V_{m r} .
\end{aligned}
$$

Eigenvalues of the SRIP system are [0 $10.3650-9.5021$ $-0.3033]$. Two poles of the SRIP system are in right half of $s$-plane; therefore, the open-loop SRIP system is unstable.

\section{Controller design}

\subsection{Swing-up control}

An energy-based swing-up controller is developed for swinging up the pendulum at its vertical downward position. The total energy required for the pendulum's upright position given by energy equation is

$$
E=\frac{1}{2} J_{e q}\left(\dot{\alpha}_{1}\right)^{2}+m g l\left(\cos \alpha_{1}+1\right)
$$

Differentiating with respect to time, this equation becomes

$$
\dot{E}=J_{e q} \dot{\alpha}_{1} \ddot{\alpha}_{1}-m g l \dot{\alpha}_{1} \sin \alpha_{1} .
$$

A non-linear controller that swings the pendulum up to achieve a given reference energy $E_{r}$ has the following form:

$$
u=\left(E-E_{r}\right) \cos \alpha_{1} \dot{\alpha}_{1} .
$$

However, to change the energy quickly the magnitude of the control signal must be fairly large. Hence, a tunable gain $\mu$ is multiplied to this equation [21]:

$$
u=\mu\left(\left(E-E_{r}\right) \cos \alpha_{1} \dot{\alpha}_{1}\right), \quad \mu>1 .
$$

This input should be bounded within system limits. Hence, a saturation function is used. Thus

$$
u=\operatorname{sat}_{u_{\max }} \mu\left(\left(E-E_{r}\right) \cos \alpha_{1} \dot{\alpha}_{1}\right),
$$

where $s a t_{u_{\max }}$ denotes a linear function that saturates at $u_{\max }$.

\section{$4.2 S M C$}

The linear model of SRIP system is valid up to $10^{\circ}$ of pendulum angle. The SRIP system is a fourth-order system and its state space model is given as

$$
\dot{x}=A x+b u .
$$

Let the sliding surface be

$$
s=\sigma^{T} x_{e}
$$

where $\sigma^{T} \in \Re^{1 \times 4}$, and $x_{e}=\left[e_{\theta} e_{\alpha} e_{\dot{\theta}} e_{\dot{\alpha}}\right]$ is the error state vector. If $x_{d}$ is the desired state vector and $x$ is the actual state vector then $x_{e}=x-x_{d}$. Gao's power rate law, which ensures control in finite time, is

$$
\dot{s}=-k|s|^{\alpha} \operatorname{sign}(s) .
$$

Derivative of this equation gives

$$
\begin{aligned}
& \dot{s}=\sigma^{T} \dot{x}_{e} \\
& \dot{s}=\sigma^{T} \dot{x}-\sigma^{T} \dot{x}_{d} \\
& \dot{s}=\sigma^{T} A x+\sigma^{T} b u-\sigma^{T} \dot{x}_{d} .
\end{aligned}
$$

Substituting Eq. (27) in this equation gives

$$
-k|s|^{\alpha} \operatorname{sign}(s)=\sigma^{T} A x+\sigma^{T} b u-\sigma^{T} \dot{x}_{d} .
$$

Rearranging this equation gives

$$
u=-\left(\sigma^{T} b\right)^{-1}\left(\sigma^{T} A x+k|s|^{\alpha} \operatorname{sign}(s)-\sigma^{T} \dot{x}_{d}\right) .
$$

The desired vector is $x_{d}=\left[\begin{array}{llll}0 & 0 & 0 & 0\end{array}\right]$ and desired derivative vector is $\dot{x}_{d}=[0000]$. The final control law is

$$
u=-\left(\sigma^{T} b\right)^{-1}\left(\sigma^{T} A x+k|s|^{\alpha} \operatorname{sign}(s)\right) .
$$

This equation provides the necessary stabilization control input to the SRIP system.

\subsection{SMC with filter}

SMC with sign function still exhibits the chattering phenomenon because of high level switching frequency in control input signal. Hence a solution better than the sign function control design is required to reduce the chattering effect in SMC. Thus a sliding-mode control with filter (SMCF) is proposed in this paper and an SMC system with LPF is shown in figure 3.

The LPF transfer function is given

$$
Q(p)=\frac{1}{\lambda p+1}
$$

where $\lambda>0$ is a small positive time constant [22] and $p=$ $\frac{d}{d t}$ is the differential operator. The equivalent control law for $\mathrm{SMC}$ is

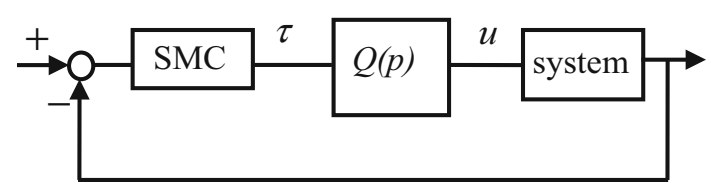

Figure 3. Sliding-mode controller with filter. 


$$
u_{e q}=\left[-\left(\sigma^{T} b\right)^{-1}\left(\sigma^{T} A x\right)\right] .
$$

If this input is applied to the system, the resultant closedloop dynamics is

$$
\dot{x}=\left[A-\left[-b\left(\sigma^{T} b\right)^{-1}\left(\sigma^{T} A\right)\right] x .\right.
$$

Closed-loop dynamics for SMC is determined by

$$
\operatorname{eig}\left[A-\left[-b\left(\sigma^{T} b\right)^{-1}\left(\sigma^{T} A\right)\right] .\right.
$$

Choice of $\lambda$ affects the closed-loop dynamics. If $0<\lambda<<$ $T s$, where $T s$ is the settling time, then effect of $\lambda$ in the closed-loop dynamics can be neglected.

The SMC with filter is aimed to drive the sliding variable ' $s$ ' to zero in the presence of system uncertainties. In figure $3, \tau$ is the sliding-mode output and $u$ is the actual control input.

The actual control input to the SRIP system is given by

$$
u=\left[-\left(c^{T} b\right)^{-1}\left(c^{T} A x+k|s|^{\alpha} \operatorname{sign}(s)\right)\right] Q(p) .
$$

This equation provides the necessary control input for a system with filter function $Q(p)$ along with SMC.

\subsection{Fuzzy logic SMC}

Fuzzy logic control has supplanted conventional techniques in many applications. A major feature of fuzzy logic is the ability to express the amount of ambiguity in individual perception and human thinking. Thus, when the mathematical model of a method does not exist, or exists but with uncertainties of disturbance, fuzzy logic control is an alternative way to deal with the unknown method. However, the large number of fuzzy rules for a high-order systems makes the research a complex problem. Therefore, significant analysis has been focussed on the FSMC.

In Eqs. (29) and (34), sign(s) is used. Sign(s) has a hard nonlinearity, which results in chattering. To eliminate chattering, $\operatorname{sign}(s)$ is replaced by a fuzzy system with smooth input and output characteristics. Values of ' $s$ ' and its derivative ' $\dot{s}$ ' are given as input to the fuzzy system. Its output is used instead of $\operatorname{sign}(s)$ in the control law (29).

$$
u=-\left(c^{T} b\right)^{-1}\left(\sigma^{T} A x+k|s|^{\alpha} F(s, \dot{s})\right) .
$$

The input control rules must be designed such that the actual path of the states always turns towards and does not cross the switching surface in phase plane to satisfy the existence condition.

In this paper, universe of both the inputs is divided into seven linguistic variables (NB - negative big, NM negative medium, NS - negative small, Z - zero, PS positive small, $\mathrm{PM}$ - positive medium and $\mathrm{PB}$ - positive big). In both cases, NB is chosen as $s$-membership function with parameters [-0.5 0.5$]$. Similarly the linguistic variable PB is chosen as ' $\dot{s}$-membership function with parameters [-2 2]. Remaining variables are chosen as triangular membership functions, in such a way that $[-1,1]$ is partitioned equally. The control rules are given in table 2 and the fuzzy surface view is shown in fig-

Table 2. Fuzzy SMC rule-based matrix.

\begin{tabular}{lccccccc}
\hline & \multicolumn{7}{c}{$\dot{s}$} \\
\cline { 2 - 7 }$s$ & PB & PM & PS & Z & NS & NM & NB \\
\hline PB & PB & PB & PB & Z & Z & Z & Z \\
PM & PB & PB & PB & Z & Z & Z & NS \\
PS & PB & PB & PM & Z & Z & NS & NM \\
Z & PB & PM & PS & Z & S & NM & NB \\
NS & PM & PS & Z & Z & NM & NB & NB \\
NM & PS & Z & Z & Z & NB & NB & NB \\
NB & Z & Z & Z & Z & NB & NB & NB \\
\hline
\end{tabular}

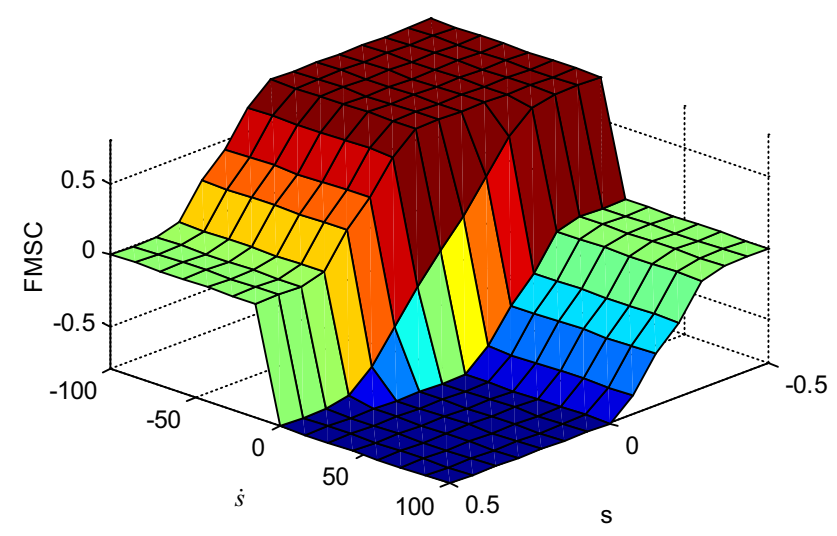

Figure 4. Fuzzy surface view.

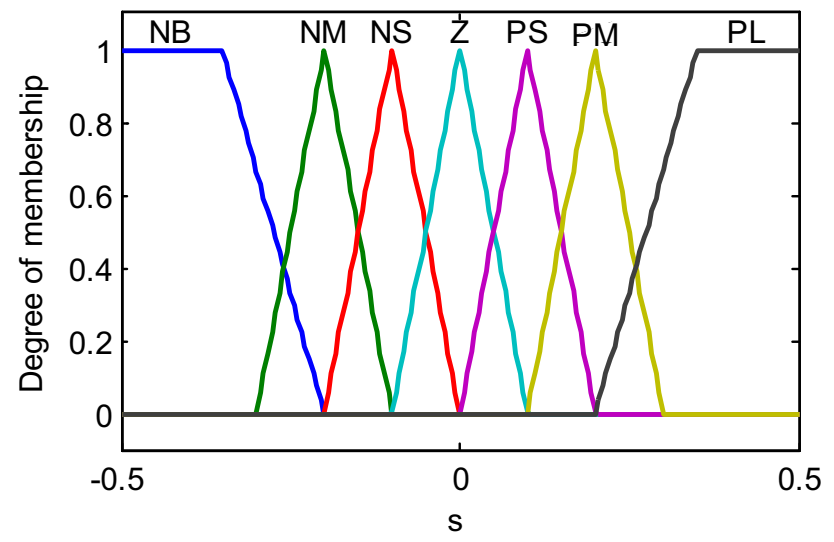

Figure 5. Fuzzy membership function of input variable $s$. 


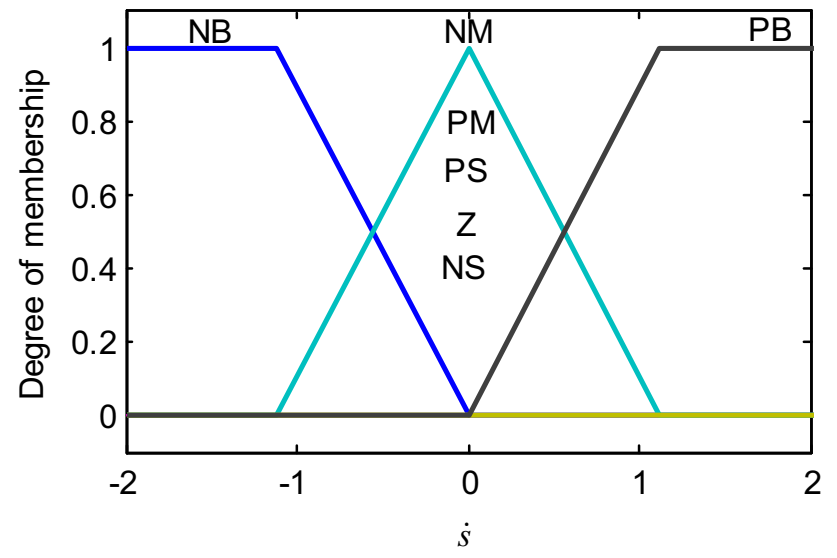

Figure 6. Fuzzy membership function of input variable $\dot{s}$.

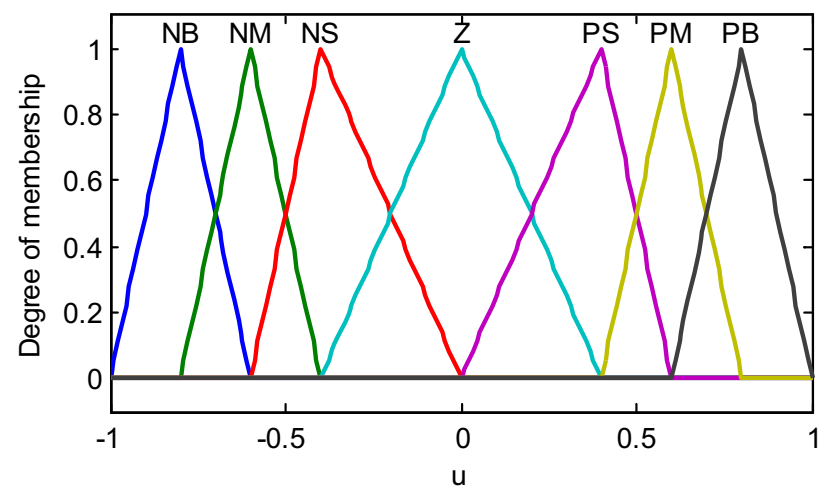

Figure 7. Fuzzy membership function of control output variable (u).

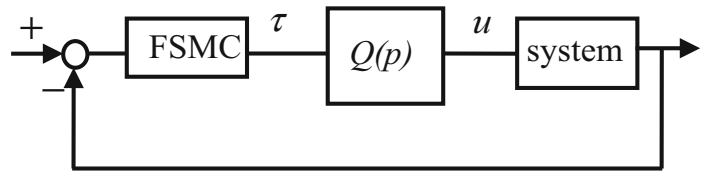

Figure 8. Fuzzy sliding-mode controller with filter (FSMCF). ure 4. The max-min composition is chosen as the fuzzy sliding-mode inference method.

The membership function of input variables sandsis shown in figures 5 and 6 and output variable ' $u$ ' is shown in figure 7 .

\subsection{Fuzzy sliding-mode control with filter}

An FSMC system with LPF is shown in figure 8.

A LPF is added with FSMC so as to improve the stabilization effect and to reduce the chattering effect of control input.

The control input to SRIP system is given by

$$
u=\left[-\left(\sigma^{T} b\right)^{-1}\left(\sigma^{T} A x+k|s|^{\alpha} F(s, \dot{s})\right] Q(p) .\right.
$$

The LPF transfer function is

$$
Q(p)=\frac{1}{\lambda p+1}
$$

where $\lambda>0$ is a small positive time constant and $p=\frac{d}{d t}$ is the differential operator.

This is a SRIP system stabilization of fuzzy sliding-mode controller with filter (FSMCF).

\section{Experimental set-up}

The SRIP stabilization control system is a typical example of integrating process. The performance of the proposed FSMC with and without filter is verified on a SRIP system. The experiments were carried out using the Quanser Q8 hard-ware-in-the loop (HIL) Interface board as shown in figure 9. The Quanser Q8 DAQ board interfaces the SRIP to the PC through a USB port as shown in figure 10 . Implementation of the proposed FSMC with and without filter is performed. The real-time workshop and real-time windows target generate the corresponding $\mathrm{C}$ code using the QuaRC software. The Quanser Q8 board acts as an intermediary for two-way data flow from the physical SRIP system to the QuaRC model. A high-resolution encoder is used for position sensing of the SRIP system.

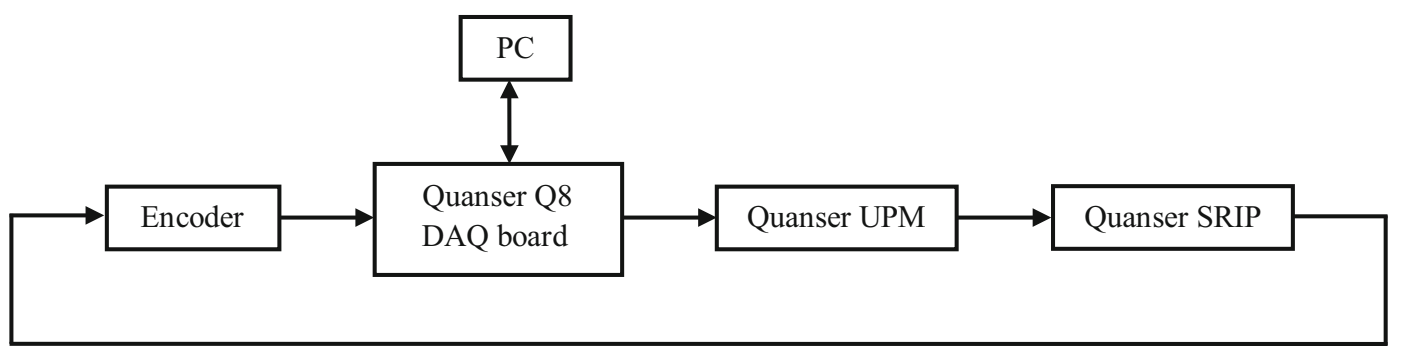

Figure 9. Block diagram of Quanser SRIP system with its interface. 


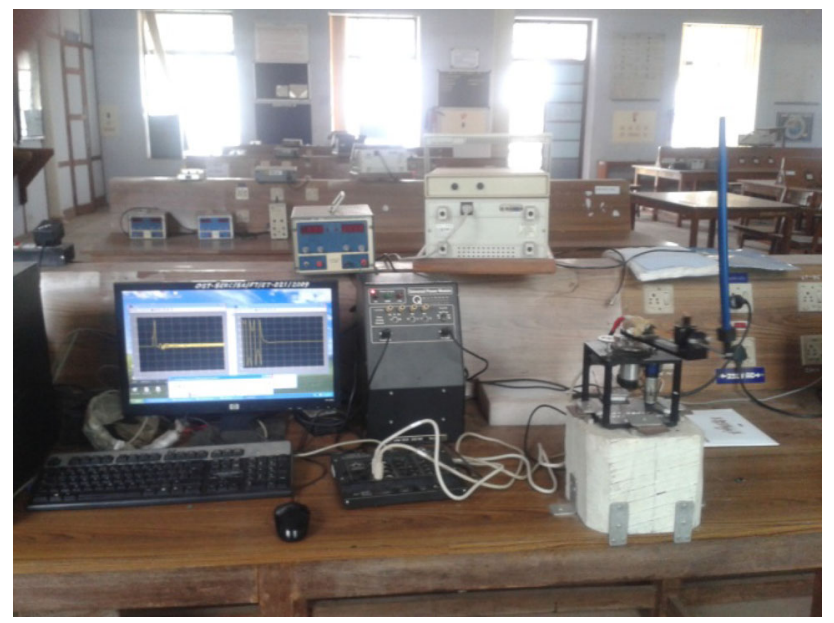

Figure 10. Experimental set-up of SRIP system.

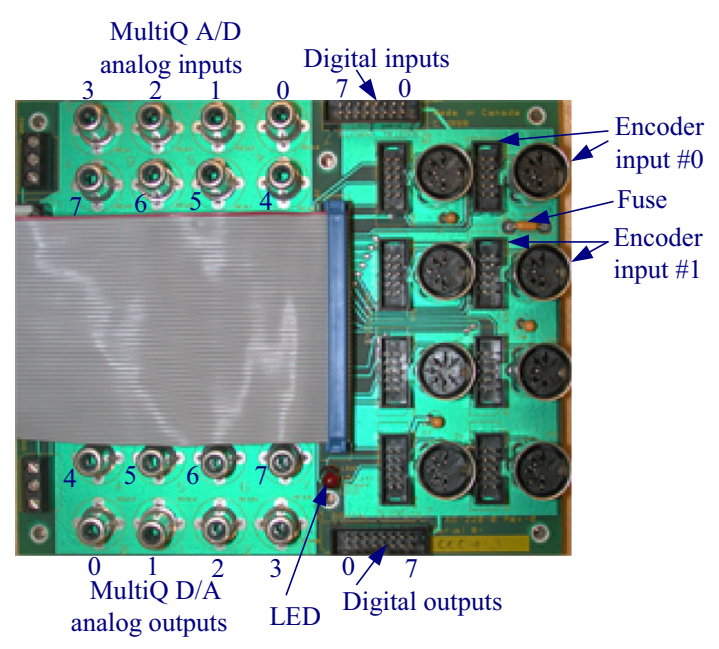

Figure 11. The Quanser Q8 DAQ board.

\subsection{Quanser Q8 DAQ board and Universal Power Module}

The Q8 is a versatile and powerful measurement control board with an extensive range on input and output support. A wide variety of devices with analog and digital sensors as well as quadrature encoders are easily connected to the Q8. This single board solution is ideal for use in control systems and complex measurement applications. Some of the salient features of Quanser Q8 DAQ board are high resolution, 14-bit inputs, high-speed sampling up to $350 \mathrm{kHz}$, simultaneous sampling of $\mathrm{A} / \mathrm{D}$, digital and encoder inputs, extensive I/O, 8 analog and digital I/O, encoders and 32 digital I/O on the same board, integrated with Matlab/ simulink/RTW via Quanser's QuaRC and PWM outputs on-board as shown in figure 11.

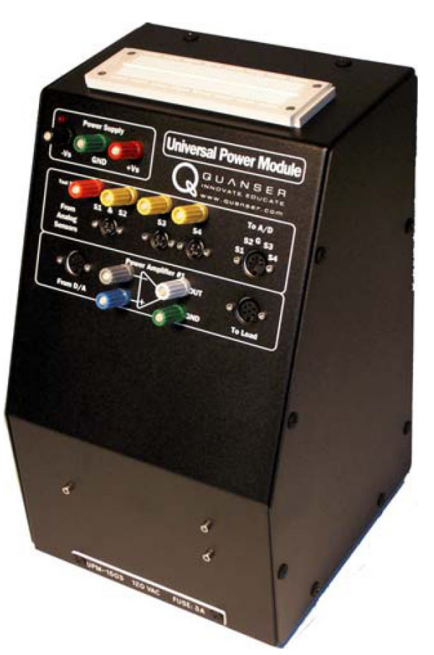

Figure 12. The Universal Power Module UPM 1503.

The Quanser Universal Power Module (UPM) is a power amplifier that is designed to drive the actuators of various Quanser experiments. Each servomotor requires a power amplifier to drive it. The power module consists of a regulated dual output $\pm 12 \mathrm{~V}_{\mathrm{DC}}$ power supply and a linear power operational amplifier as shown in figure 12. The top section labelled DC Power Supply has three binding posts set at $\pm 12 \mathrm{~V}_{\mathrm{DC}}$ with maximum current rating of $1 \mathrm{~A}$. The power section is powered via a differential power supply with the following ratings: maximum power output $45 \mathrm{~W}$, maximum current output $3 \mathrm{~A}$, maximum output voltage $15 \mathrm{~V}$, power bandwidth $60 \mathrm{kHz}$, small signal bandwidth $700 \mathrm{kHz}$ and slew rate $9 \mathrm{~V} / \mu \mathrm{s}$. The power modules have several connectors, each referred to as a Quick Connect System. The output of the DAQ is connected to the power amplifier input. The signal from the analog sensor is connected to the analog inputs. The output of the power amplifier labelled to motor is connected to the servomotor terminals. Sampling period is $1 \mathrm{~ms}$.

\section{Results and discussion}

Initially the pendulum is at downward position $\alpha_{1}=\pi$ when the plant is started. Energy-based swing-up control rises the pendulum arm towards upright position. When ' $\alpha$ ' reaches within $\pm 15^{\circ}$, stabilization control is activated. This has been used to bring the pendulum in the linear region, wherein the linear model is valid for the control development. Further, in the linear region, a stabilization control has been adopted. Closed-loop eigenvalues are chosen to be $\left[\begin{array}{llll}0 & -5.0623+3.2067 & -5.0623-3.2067 & -5.7643\end{array}\right]$. These values are chosen based on our experience with the plant such that the dynamics is within the system limits. From the values of eigenvalues, the sliding surface obtained is $\sigma^{T}=\left[\begin{array}{lll}17.076 & -76.9016 & 7.3774-12.1598\end{array}\right] x, k=10$ and 

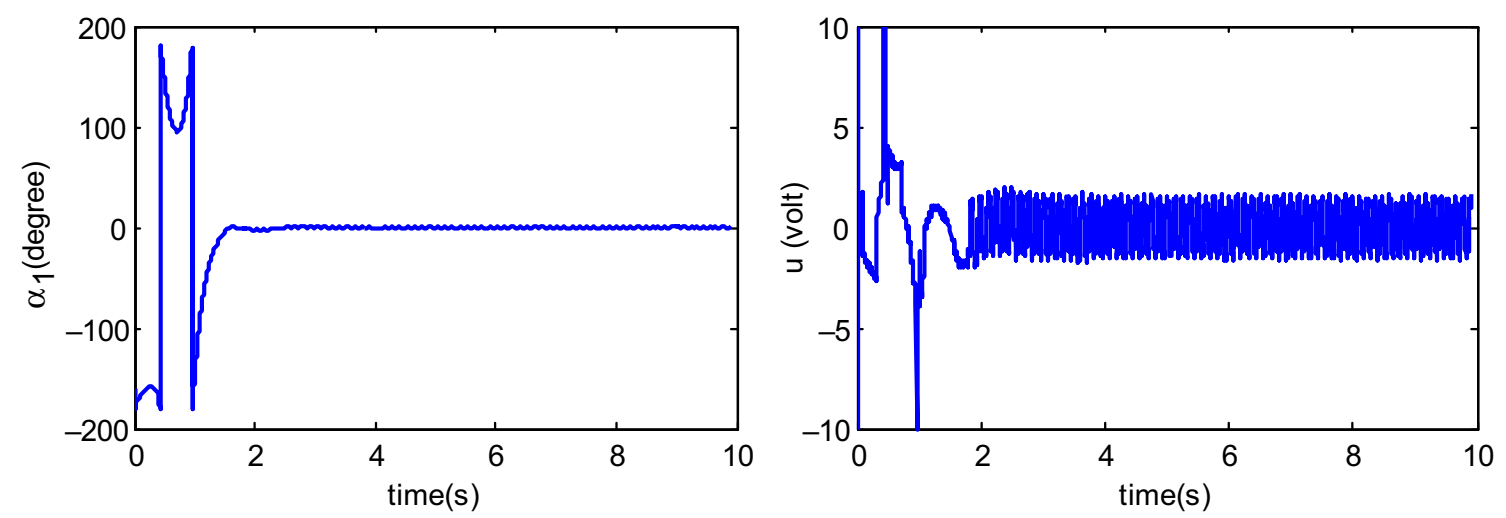

Figure 13. Sliding-mode control response.
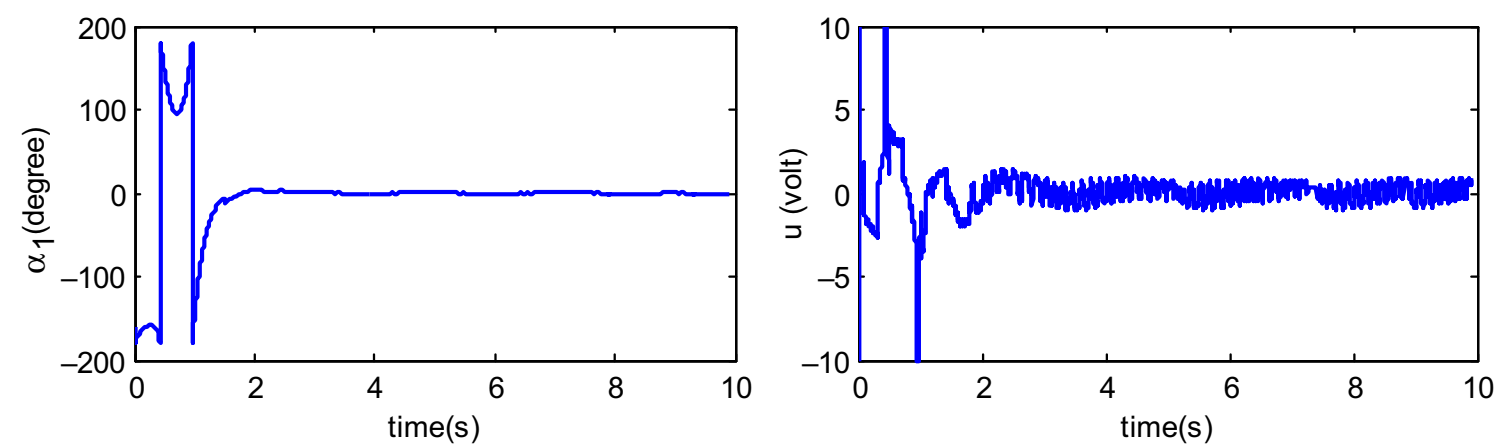

Figure 14. Sliding-mode control response with filter $(\lambda=0.001)$.
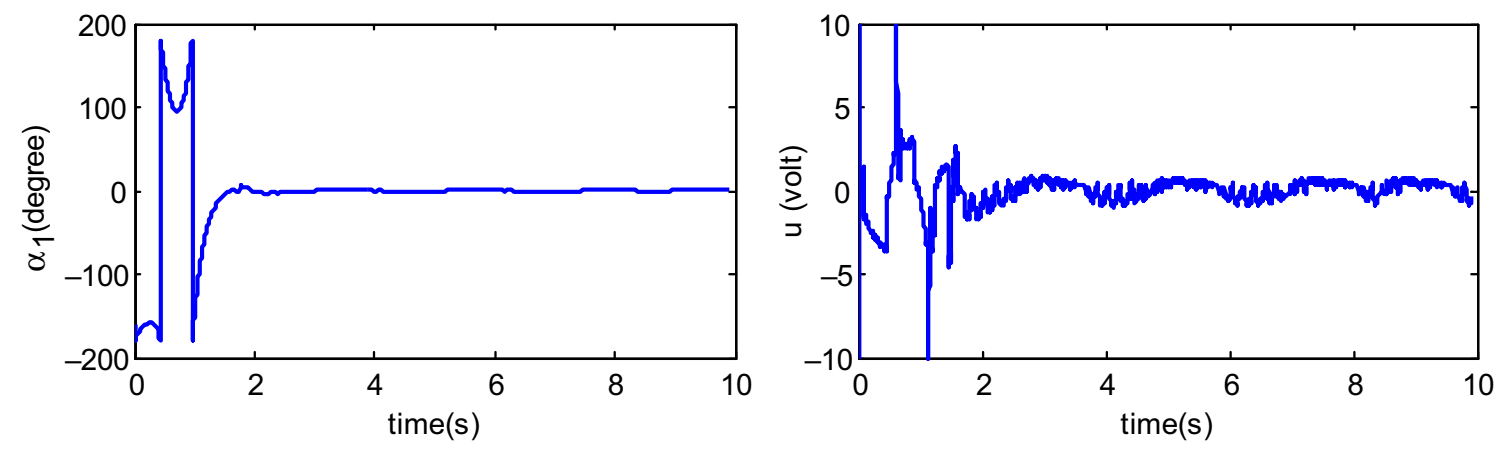

Figure 15. Sliding-mode control response with filter $(\lambda=0.002)$.

$\alpha=0.3$. A continuous sinusoidal disturbance of magnitude $5^{\circ}$ and frequency $1 \mathrm{rad} / \mathrm{s}$ is used to test the disturbance rejection capability.

The performance of SMC is as shown in figure 13. The pendulum swings at least one time before stabilization at $0^{\circ}$. Chattering effect in the control variable is observed for the SMC. The sign function used in SMC is not able to alleviate the chattering.
Without filter, the settling time is on the order of $2 \mathrm{~s}$. The selection of $\lambda$ as less than the settling time is $0.001 \mathrm{~s}$. Hence closed-loop dynamics will not be affected. The time constants of the LPF $(\lambda)$ is varied from 0.001 to 0.003 in steps. Figures 14,15 and 16 show the effect of filtered SMC. It is found that there is no appreciable variation in the dynamics of pendulum angle. However, chattering effect is not completely reduced when compared with SMC with filter response. 

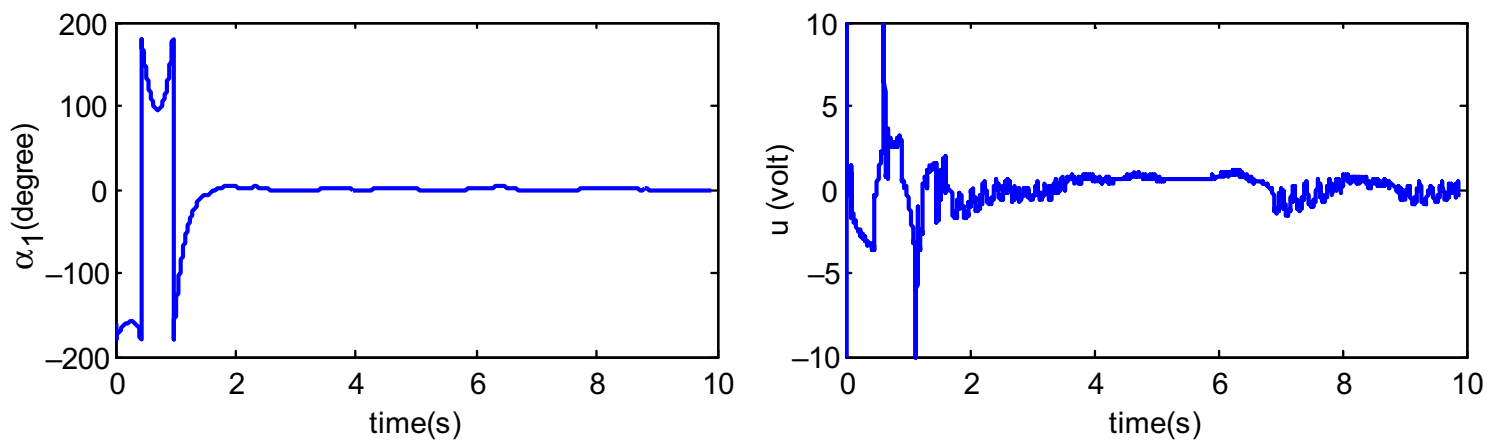

Figure 16. Sliding-mode control response with filter $(\lambda=0.003)$.
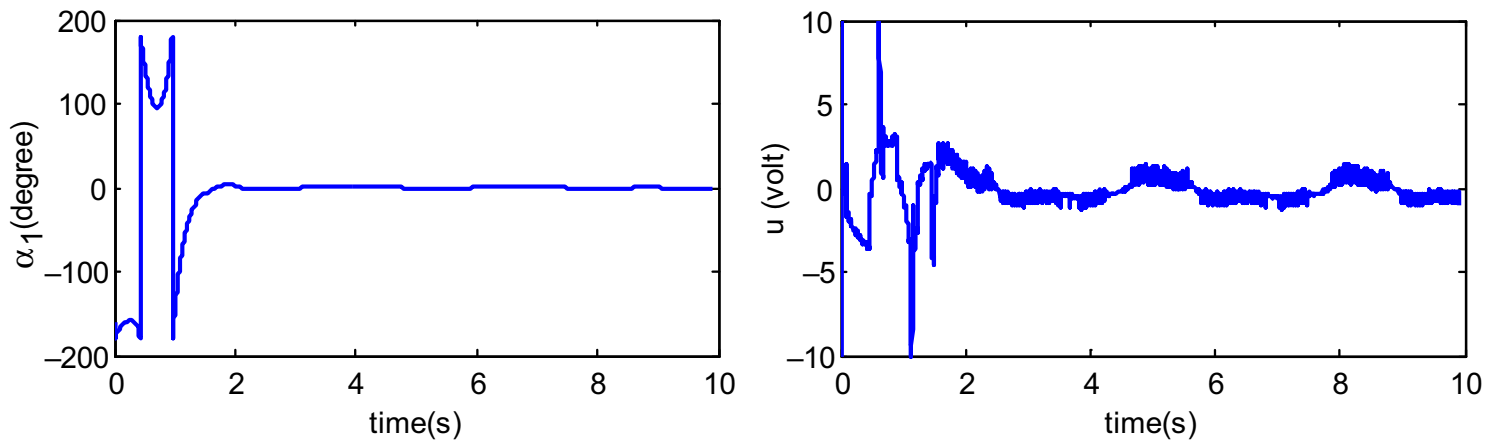

Figure 17. Fuzzy sliding-mode controller without filter.
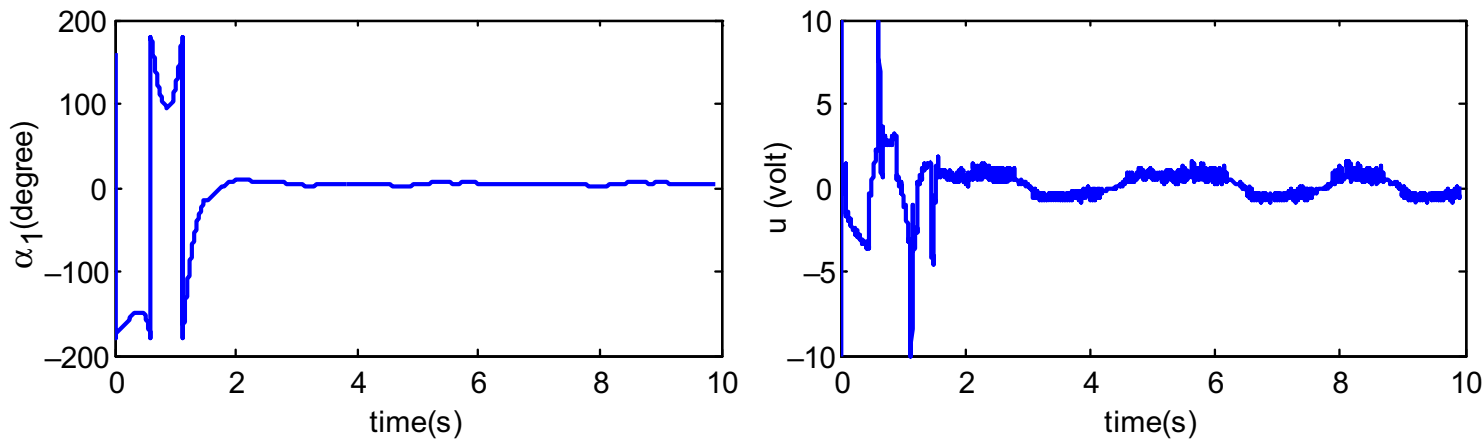

Figure 18. Fuzzy sliding-mode controller with filter $(\lambda=0.001)$.

The response of SRIP stabilization using FSMC without filter is shown in figure 17. There is a slight improvement in the reduction of chattering effect for FSMC without filter.

A similar attempt is made by varying the time constant of the filter from 0.001 to 0.003 in steps to the FSMCF and the results are shown in figures 18, 19 and 20. It is found that there is no appreciable change in the closed-loop dynamics. However, chattering effect is reduced to a great extent when compared with FSMC with filter response. In all cases (SMCF and FSMCF) there is no chattering effect in control input. However, it is observed that FSMCF response is better than that of SMCF for the control input.

The frequency response of SMC and FSMC with filter controllers using fast Fourier Transform (FFT) is obtained. The performance curves of $\alpha$ (output signal) for SMC and FSMC with filter are as shown in figure 21. The response curve of FSMC with filter is better than that of 

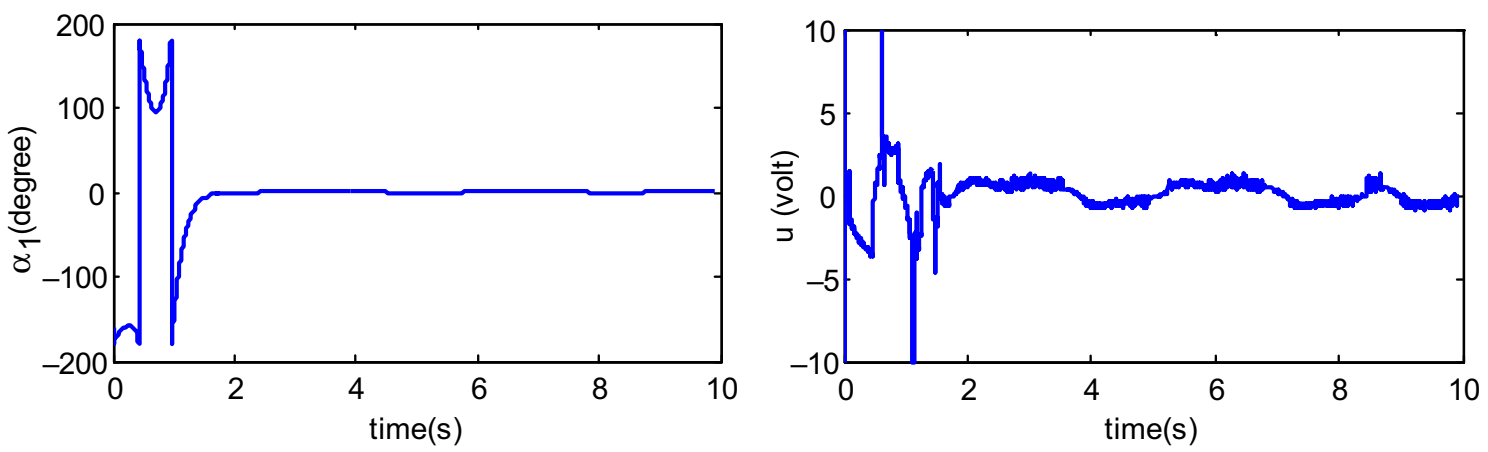

Figure 19. Fuzzy sliding-mode controller with filter $(\lambda=0.002)$.
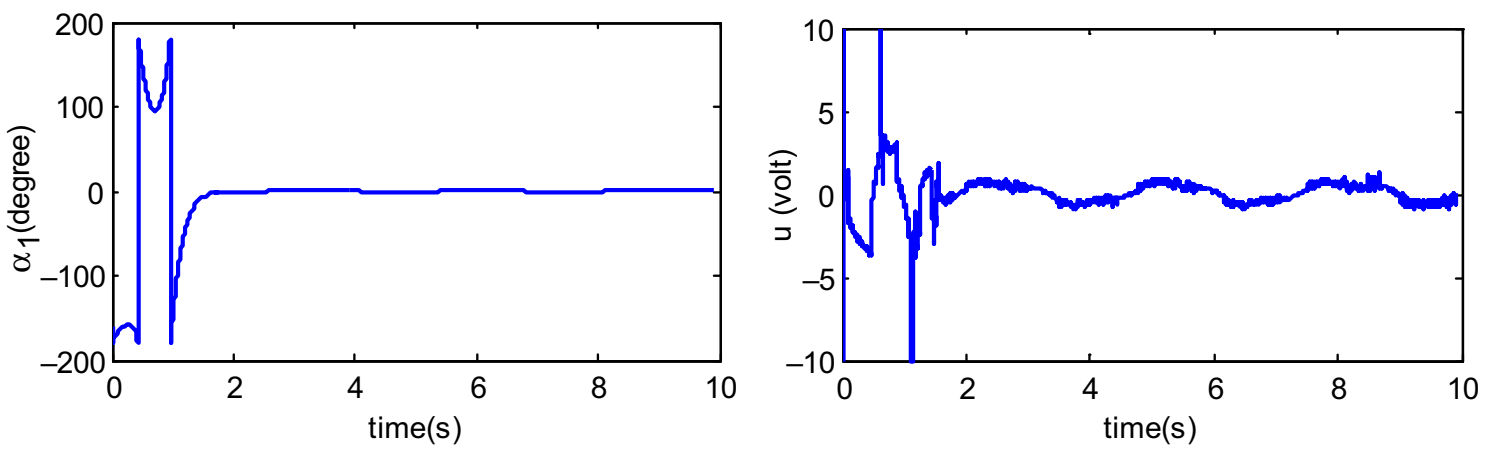

Figure 20. Fuzzy sliding-mode controller with filter $(\lambda=0.003)$.

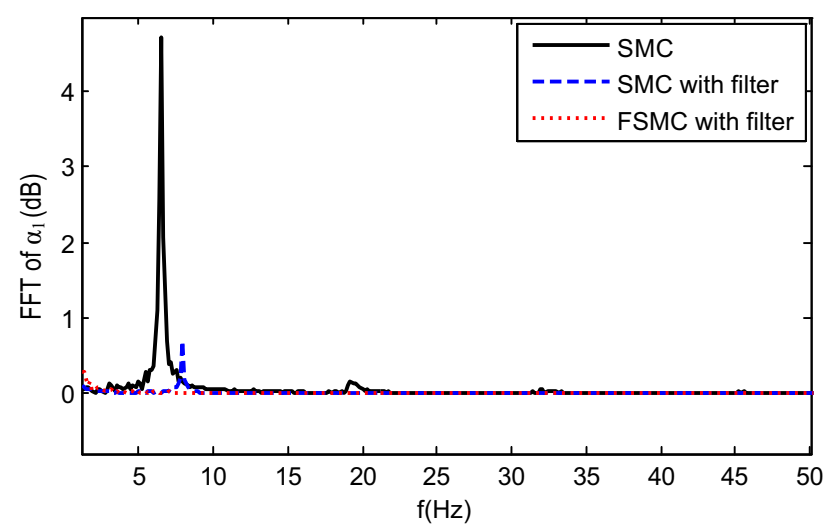

Figure 21. Comparison of SMC, SMC with filter and FSMC with filter for output signal in frequency domain $(\lambda=0.003)$ using FFT.

SMC with and without filter and it reduces the magnitude level.

The performance curves of the SMC, SMC with filter and FSMC with filter of $u$ (control input) are obtained using FFT and shown in figure 22. It is observed that the response curve of the FSMC with filter is better than that of SMC with filter and it reduces the magnitude level. Thus it is evident that FSMC with filter has less overshoot and undershoot compared with SMC with filter.

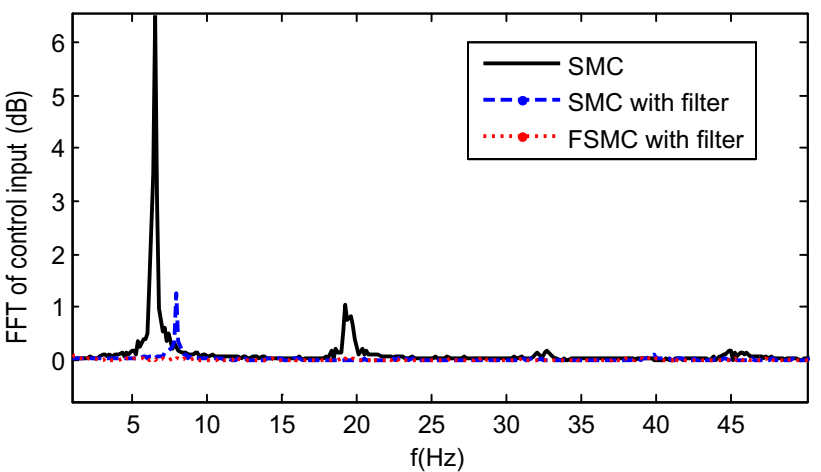

Figure 22. Comparison of SMC, SMC with filter and FSMC with filter for input signal in frequency domain $(\lambda=0.003)$ using FFT.

\section{Conclusion}

Elimination of chattering in FSMC using input filter is proposed in this paper. The proposed methodology is validated experimentally in a real-time SRIP set-up. Response and control input for SMC, FSMC with and without filter for various filter time constants are presented. FFTs of the control input for these cases are compared. Results indicate that input filtering of FSMC reduces the effect of chattering to a great extent. In this paper, SMC and FSMC are 
designed only for linear operating region. However, the proposed method can easily be extended to nonlinear cases.

\section{Acknowledgements}

This work is supported in part by DST-FAST Track Project (SR/FT/ET-021/2009) from Government of India.

\section{Appendix}

See table 3.

Table 3. Parameters of the SRIP.

\begin{tabular}{lcc}
\hline Symbol & Description & $\begin{array}{c}\text { Nominal value (SI } \\
\text { units) }\end{array}$ \\
\hline$\eta_{g r}$ & Gearbox efficiency & 0.9 \\
$\eta_{m r}$ & $\quad$ Motor efficiency & 0.69 \\
$B_{e q}$ & Equivalent viscous damping & 0.0040 \\
\multicolumn{3}{c}{ coefficient } \\
$J_{e q}$ & Equivalent moment of inertia at \\
$K_{g r}$ & $\quad$ the load \\
$K_{m r}$ & Total gear ratio (kg) & 0.0021 \\
$K_{t}$ & Motor torque constant $(\mathrm{Nm} / \mathrm{A})$ & 70 \\
$L$ & Length of pendulum $(\mathrm{m})$ & 0.0077 \\
$m$ & Pendulum mass $(\mathrm{kg})$ & 0.0077 \\
$J_{m r}$ & Motor inertia (kg m $\left.{ }^{2}\right)$ & 0.1683 \\
$R_{m r}$ & Motor armature resistance $(\Omega)$ & $3.87 \mathrm{e}-7$ \\
\hline
\end{tabular}

\section{References}

[1] Astrom K J and Furta K 2000 Swing up a pendulum by energy control. Automatica 36(2): 287-295

[2] Akhtaruzzaman M and Shafie A A 2010 Modeling and control of a rotary inverted pendulum using various methods, comparative assessment and result analysis. In: Proceedings of the IEEE International Conference on ICMA, pp. $1342-1347$

[3] Hung J Y, Gao W B and Hung J C 1993 Variable structure control: a survey. IEEE Trans. Ind. Electron. 40: 2-22

[4] Chen C K, Lin C J and Yao L C 2004 Input-state linearization of rotary inverted pendulum. Asian J. Control 6(1): $130-135$

[5] Liu H 2013 Adaptive fuzzy sliding mode control for $X-$ $Z$ inverted pendulum. J. Theor. Appl. Inf. Technol. 49(3): 896-900
[6] Lin C M and Mon Y J 2005 Decoupling control by hierarchical fuzzy sliding mode controller. IEEE Trans. Control Syst. Technol. 13(4): 593-598

[7] Yi J, Yubazaki N and Hirota K 2001 Upswing and stabilization control of inverted pendulum system based on the SIRMs dynamically connected fuzzy inference model. Fuzzy Sets Syst. 122: 139-152

[8] Kurode S, Chalanga A and Bandyopadhyay B 2011 Swingup and stabilization of rotary inverted pendulum using sliding modes. In: Proceedings of the 18th world congress IFAC, pp. $10685-10690$

[9] Utkin V I 1993 Sliding mode control design principles and applications to electric drive. IEEE Trans. Ind. Electron. 40: 23-36

[10] Antic D and Dimitrijevic S 1999 One application of low-pass filter in sliding mode control system with PI action. J. Electr. Eng. 82: 33-37

[11] Sira-Ramirez H 1992 On the sliding mode control of the nonlinear systems. Syst. Control Lett. 19: 303-312

[12] Suryawanshi P V, Shendge P D and Phadke S B 2016 A boundary layer sliding mode control design for chatter reduction using uncertainty and disturbance estimator. Int. J. Dyn. Control 4(4): 456-465

[13] Lee H and Utkin V I 2007 Chattering suppression methods in sliding mode control systems. Annu. Rev. Control 31: 179-188

[14] Utkin V I and Chang H C 2002 Sliding mode control on electro-mechanical systems. Math. Probl. Eng. 8(4): 451-473

[15] Park M S and Chwa D 2009 Swing-up and stabilization control of inverted-pendulum systems via coupled slidingmode control method. IEEE Trans. Ind. Electron. 56(9): 3541-3555

[16] Park K B and Lee J J, 1997 Sliding mode controller with filtered signal for robot manipulators using virtual plant/controller. Mechatronics 7(3): 277-286

[17] Batolini G and Pydynowski P 1996 An improved chattering free V.S.C. scheme for uncertain dynamical systems. IEEE Trans. Autom. Control 41: 1220-1226

[18] Chen C C and Chen M S 2008 A new design for chattering reduction in sliding mode control. In: Proceedings of the 17th World Congress IFAC, pp. 9877-9881

[19] Tseng M L and Chen M S 2010 Chattering reduction of sliding mode control by low pass filtering the control signal. Asian J. Control 12(3): 392-398

[20] Ramesh R, Balamurugan S and Venkatesh P 2013 Real time servo motor control of single rotary inverted pendulum using dSPACE. Int. J. Soft Comput. Eng. 3(2): 2231-2307

[21] Benjanarsuth T and Nundrakwang S 2008 Hybrid controller for rotational inverted pendulum systems. In: Proceedings of the SICE Annual Conference, pp. 1889-1894

[22] Utkin V, Guldner J and Shi J 2009 Sliding mode control in electro-mechanical systems, 2nd ed. New York: CRC Press 